\title{
Spinodally synthesized magnetoelectric
}

\author{
Shenqiang Ren and Manfred Wuttiga) \\ Department of Materials Science and Engineering, University of Maryland, College Park, Maryland 20742
}

(Received 24 May 2007; accepted 7 July 2007; published online 20 August 2007)

\begin{abstract}
Lead zirconium titanate/nickel ferrite (PZT/NFO) composites have been produced by crystallizing and spinodally decomposing a gel in a magnetic field below the Curie temperature of NFO. The gel had been formed by spinning a sol onto a silicon substrate. The ensuing microstructure, characterized by atomic force microscopy, magnetic force microscopy, (Lorentz) transmission electron microscopy, and scanning electron microscopy, is nanoscopically periodic and, determined by the direction of magnetic annealing field, anisotropic. The wavelength of the PZT/NFO alternation, $25 \mathrm{~nm}$, agrees within a factor of 2 with the theoretically estimated value. The macroscopic ferromagnetic and magnetoelectric responses correspond qualitatively and semiquantitatively to the features of the nanostructure. The maximum of the field dependent magnetoelectric susceptibility equals $1.8 \mathrm{~V} / \mathrm{cm}$ Oe. (C) 2007 American Institute of Physics. [DOI: $10.1063 / 1.2767174$ ]
\end{abstract}

Landau et al. predicted the existence of solids displaying a magnetoelectric (ME) response in $1960 .{ }^{1}$ In composite materials, this $\mathrm{ME}$ effect is realized by using the concept of product properties introduced by Van Suchetelen in 1972 . $^{2}$ The recent revival of research on MEs has resulted in impressive progress in both natural and artificial, i.e., composite, MEs. ${ }^{3-7}$ Composite MEs have been synthesized "top down" by macroscopically combining the ferromagnetic and ferroelectric components and/or by applying thin film techniques to produce multilayers. Examples are bulk composites of Terfenol-D/PVDF (Polyvinylidene Difluoride, ${ }^{8,9} \mathrm{Fe}-\mathrm{Ga} / \mathrm{PMN}-\mathrm{PT}$ (Lead magnesium niobateLead titanate), ${ }^{10} \mathrm{CoFe}_{2} \mathrm{O}_{4} / \mathrm{BaTiO}_{3},{ }^{11}$ Terfenol-D/PMN-PT ${ }^{12}$ LSMO/PZT, ${ }^{13,14}$ and Metglas/PVDF laminates. ${ }^{15,16}$ Pulsed laser films from multiphase targets can self-organize under the influence of epitaxial strains and form regular ${ }^{17,18}$ two phase ME 1-3 CFO/BTO nanocomposites. ${ }^{19-22}$ Here, we report on a ME composite formed by spinodal decomposition. In that case, only the innate thermodynamic driving force self-organizes a periodic ME nanocomposite. Decomposition in a magnetic field should yield an anisotropic laminate. This mode of synthesis was envisioned long ago ${ }^{2}$ but not pursued until now.

Stoichiometric $\quad\left[\mathrm{Pb}_{1.1}\left(\mathrm{Zr}_{0.53} \mathrm{Ti}_{0.47}\right) \mathrm{O}_{3}\right]_{50} /\left[\mathrm{NiFe}_{2} \mathrm{O}_{4}\right]_{50}$ [lead zirconate titanate (PZT)/nickel femite (NFO)] thin films were prepared by thermal decomposition of mixed metal polymeric precursor gels followed by spin coating onto Au-electroded silicon wafers and baking. The polymeric precursor solution was prepared using the Pechini method as described by Calzada and Milne. ${ }^{23}$ The approximately $200 \mathrm{~nm}$ thick films were patterned, magnetoannealed below the Curie temperature of NFO, and rapidly cooled to room temperature in zero field.

Structural characterization was carried out by scanning X-ray microdiffraction using a D8 DISCOVER Bruker-AXS. Thermal properties were studied by differential thermal and thermogravimetric analyses. Transmission electron microscopy (TEM), including Lorentz TEM, images and selected area diffraction patterns were obtained with a JEOL 4000-FX

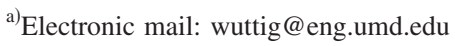

and a JEOL 2100F emission TEM. Polarization values were determined with a RT 66A Radiant Technologies ferroelectric loop tracer. A superconducting quantum interference device magnetometer, vibrating sample magnetometer, and magnetic force microscopy were used to perform magnetic characterization. Local compositions were determined with the use of a JEOL JSM-6500F secondary electron emission spectrometer.

$\mathrm{X}$-ray diffraction measurements confirmed that the composite consists of perovskite and spinel. The ferroelectric and ferromagnetic Curie temperatures of the composite were $20{ }^{\circ} \mathrm{C}$ below the values of NFO and PZT. Hence, the chemical compositions of the PZT and NFO components almost equal that of the "pure" components. This will justify the use of known properties of PZT and NFO in theoretical estimations. Results of microscopic characterizations are presented in Figs. 1 and 2 displaying structural and compositional information of a film annealed in fields of $0.6 \mathrm{~T}$ directed parallel and perpendicularly to the plane of the film. The images show how the microstructure evolved in the course of the annealing process. Initially, after a short annealing of $2 \mathrm{~h}$ at $460{ }^{\circ} \mathrm{C}$, the structure is dense but near amorphous, as can be seen from the electron diffraction pattern inset of Fig. 1(a). At longer annealing times of $12 \mathrm{~h}$ at $480{ }^{\circ} \mathrm{C}$, a striped pattern appears. The electron diffraction inset in Fig. 1(b) indicates that the composite is now polycrystalline, as can also be inferred from the ferroelectric and ferromagnetic characterizations to be discussed below. All microstructures are periodic, as indicated by the Fourier transform insets. The transforms indicate the existence of decomposition waves with a wavelength of approximately $25 \mathrm{~nm}$. The same periodicity can be seen in the Fourier transform of a Lorentz TEM pointing out that one component of the ME composite is ferromagnetic, as also seen in magnetic force microscopy images. Figure 1(b) also demonstrates that the wave vector $\mathbf{k}$ of the stationary decomposition wave is aligned parallel to the annealing field $\mathbf{H}_{a}$. The elemental composition analyses confirm that the periodic stripes are composed of NFO and PZT, respectively (see Fig. 2). This figure indicates that the chemical composition of the film alternates as it would in a spinodally decomposed solid. The power density in the upper 

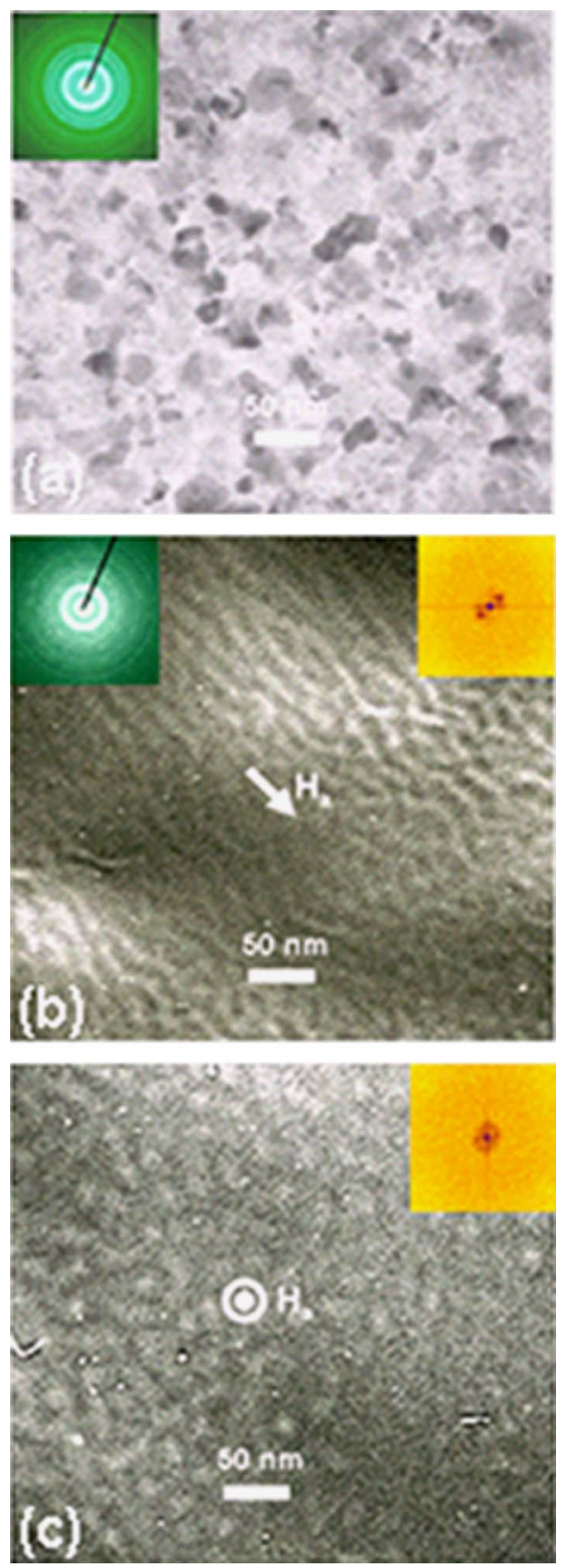

FIG. 1. (Color online) (a) TEM image of PZT/NFO sample annealed $2 \mathrm{~h}$ at $460{ }^{\circ} \mathrm{C}$. The inset shows the diffraction pattern of the as-spun amorphous state of the composite. (b) TEM image of a PZT/NFO sample annealed $12 \mathrm{~h}$ at $480{ }^{\circ} \mathrm{C}$, i.e., below the Curie temperature of NFO, in an in-plane magnetic field $H_{a}=0.6 \mathrm{~T}$ indicated by the arrow. The inset represents the diffraction pattern of the displayed structure. (c) Same as (b) but annealed in an out-of-plane magnetic field $H_{a}=0.6 \mathrm{~T}$.

right points to two standing decomposition waves that are mutually shifted by $\frac{1}{2} \lambda$, as expected.

The images of the phase-separated film have the appearance of spinodal decomposition. Two kinds of composition waves appear as a solid decomposes in a magnetic field: ${ }^{24}(1)$ waves whose wavelength is affected by the ferromagnetic nature of the solid, termed "parallel waves," and (2) waves whose wavelength is additionally affected by the magnetic poles that form upon decomposition, termed "perpendicular waves." The present experiment favors parallel waves whose wavelength is determined by parameters that are mostly unavailable. For large undercooling, as is the case in this experiment, ${ }^{25}$ the wavelengths of the parallel and perpen-

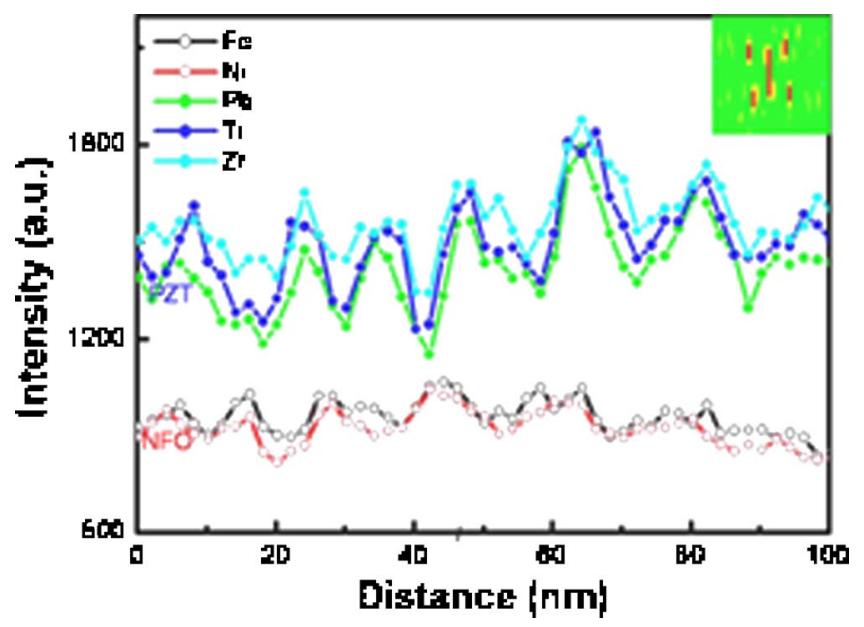

FIG. 2. (Color online) Standing compositional waves in the PZT/NFO composite. The noise of this compositional analysis does not permit statements about the exact composition of the PZT and NFO regions. However, it can be seen that the concentration of the two groups of elements, $\mathrm{Pb}, \mathrm{Zr}$, Ti, and $\mathrm{Ni}, \mathrm{Fe}$, forming the PZT and NFO components of the composite is maximized in a periodic fashion. The power Fourier transform displayed at the upper right indicates the existence of two waves. The four maxima indicate their mutual phase shift of $\frac{1}{2} \lambda$.

dicular waves approach each other. Hence, the wavelength of perpendicular waves will be estimated since the magnetic parameters controlling them are available. The ensuing wavelength $\lambda_{t}$ would thus read $\left(2 \pi / \lambda_{t}\right)^{2} \approx\left[\partial^{2} S / \partial c^{2}\left(T_{s}-T\right)\right.$ $\left.+\pi(\partial M / \partial c)^{2}\right] / 2(K+A),{ }^{24}$ where $A$ is the exchange energy. It can be estimated by neglecting the derivative of the entropy $s$ and the gradient energy $K$. By approximating further to finite differences and focusing on the observed structure, $\Delta M^{2}=\mu_{0} M_{T}^{2}$ and $\Delta c=1, M_{T}$ being the saturation magnetization of NFO at the annealing temperature, it follows that $\lambda_{t}^{2} \approx 8 \pi\left(A / M_{T}^{2}\right)$. Inserting into this expression $A^{\mathrm{NFO}} \approx 6$ $\times 10^{-7} \mathrm{erg} \mathrm{cm}^{-1}$ and $M_{T}=1 \mathrm{emu} / \mathrm{cm}^{3}, \lambda_{t} \approx 12 \mathrm{~nm}$. This length equals about one-half of the observed wavelength, $\lambda$ $\approx 25 \mathrm{~nm}$ [see Fig. 1(b)]. In view of the approximations made, the difference between $\lambda_{t}$ and $\lambda$ is not unexpected.

The macroscopic properties of the ME PZT/NFO composite agree qualitatively and quantitatively with the microstructure shown in Fig. 1(b). The PZT/NFO composite is magnetically hard and soft when magnetized in the direction perpendicular and parallel to the stripe pattern. The difference of the anisotropy energies in both directions, $\Delta K_{\text {exp }}$ $=0.2 \times 10^{6} \mathrm{ergs} / \mathrm{cm}^{3}$, equals the difference of the demagnetization energies in those directions, $\frac{1}{2} M_{s}^{2}\left(N_{\perp}-N_{\|}\right)$. With $M_{s}^{\mathrm{NFO}}=265 \mathrm{emu} / \mathrm{cm}^{3}$ and $\left(N_{\perp}-N_{\|}\right)=2 \pi, \Delta K_{t} \approx \pi M_{s}^{2}=0.22$ $\times 10^{6} \mathrm{ergs} / \mathrm{cm}^{3}=1.1 \Delta K_{\text {exp }}$, signaling agreement.

The ME coupling between the ferroelectric PZT and ferromagnetic NFO components is elastically mediated, that is, the $\mathrm{ME}$ susceptibility $\alpha_{H}=\left(\partial P^{\mathrm{PZT}} / \partial \sigma^{\mathrm{NFO}}\right)\left(\partial \sigma^{\mathrm{NFO}} / \partial H\right){ }^{2}$ Here, the magnetic field was applied perpendicular and parallel to the PZT/NFO stripelike pattern structure depicted in Fig. 3, lower inset. The negative magnetostriction of NFO shrinks the PZT, thereby decreasing its polarization by -8 and $-3 \mu \mathrm{C} / \mathrm{cm}^{2}$ at $10 \mathrm{kOe}$ for the two cases where $\mathbf{H} \| \mathbf{k}$ and $\mathbf{H} \perp \mathbf{k},|\mathbf{k}|=2 \pi / \lambda$. The saturation is attributed to that of the magnetostriction of NFO. The expected magnetically induced polarization of PZT can be estimated as

$$
\Delta P_{\mathrm{i}, t}^{\mathrm{PZT}} \approx \frac{4 \lambda^{\mathrm{NFO}} d_{33}^{\mathrm{PZT}}}{1 / E^{\mathrm{PZT}}+1 / E^{\mathrm{NFC}}+2 / E^{\mathrm{SiO} O_{2}}}
$$




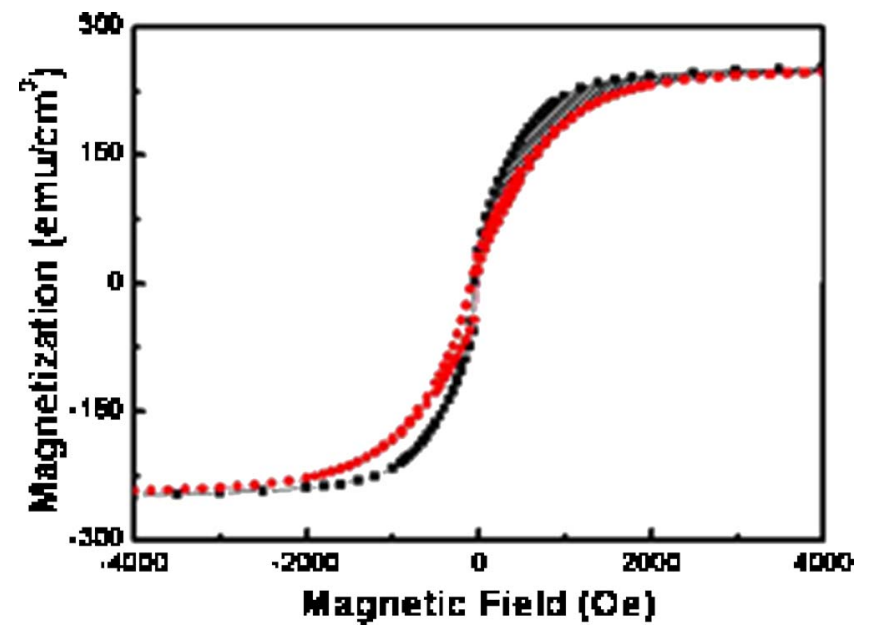

FIG. 3. (Color online) ME characteristic and ME susceptibilities (upper inset) of a PZT/NFO film composite annealed for $12 \mathrm{~h}$ at $480{ }^{\circ} \mathrm{C}$ in an in-plane magnetic field $H_{a}=0.6 \mathrm{~T}$. red line and stars: $\mathbf{H}_{a} \perp \mathbf{H} \| \mathbf{k}$; black line and open circles: $\mathbf{H}_{a} \perp \mathbf{H} \| \mathbf{k}$. The inset on the lower right indicates the placement of the electrodes

$$
\Delta P_{\perp, t}^{\mathrm{PZT}} \approx \frac{1}{4}\left(E^{\mathrm{PZT}}+E^{\mathrm{NFO}}+2 E^{\mathrm{SiO}_{2}}\right) \lambda^{\mathrm{NFO}} d_{13}^{\mathrm{PZT}} .
$$

$\lambda^{\mathrm{NFO}}$ is the magnetostriction constant of NFO. Note that $\Delta P$ is independent of the wavelength. However, the magnitude of the wavelength does affect the total charge that is accumulated at the electrodes. With $E^{\mathrm{PZT}}=60 \mathrm{GPa}, d_{33}^{\mathrm{PZT}}$ $=250 \mathrm{pC} / \mathrm{N}, \quad d_{13}^{\mathrm{PZT}}=125 \mathrm{pC} / \mathrm{N}, \quad \lambda^{\mathrm{NFO}}=-33 \mathrm{ppm}, \quad E^{\mathrm{NFO}}$ $=200 \mathrm{GPa}^{26}$ and $E^{\mathrm{SiO}_{2}}=60 \mathrm{GPa},{ }^{27} \Delta P_{\|, t}^{\mathrm{PZT}}=-6 \mu \mathrm{C} / \mathrm{cm}^{2}$ and $\Delta P_{\perp, t}^{\mathrm{PZT}}=-3.9 \mu \mathrm{C} / \mathrm{cm}^{2}$. Both expected polarizations agree within $30 \%$ with the experimentally observed values (see Fig. 3). Using the maximum value of the derivative $d \lambda^{\mathrm{NFO}} / d H,{ }^{28}$ the theoretical estimates for the ME susceptibilities become $\alpha_{H \|, t}(\mathrm{PZT} / \mathrm{NFO})=2.37 \mathrm{~V} / \mathrm{cm} \mathrm{Oe}$ and $\alpha H_{\perp, t}(\mathrm{PZT} / \mathrm{NFO})=2.4 \mathrm{~V} / \mathrm{cm}$ Oe. Differentiating the experimentally observed $\Delta P^{\mathrm{PZT}}=f(H)$ yields maxima $\alpha_{H \|}(\mathrm{PZT} / \mathrm{NFO})=1.5 \mathrm{~V} / \mathrm{cm} \mathrm{Oe} \quad$ and $\quad \alpha_{H \perp}(\mathrm{PZT} / \mathrm{NFO})$ $=1.8 \mathrm{~V} / \mathrm{cm} \mathrm{Oe}$, see upper inset of Fig. 3, corresponding approximately to the above estimates and substantially higher than achieved by ceramic technologies. ${ }^{29}$ In general, the ME susceptibility is a tensor. ${ }^{30}$ Only two of its components are presented here.

In summary, this letter demonstrates that MEs can be synthesized by spinodal decomposition. The observed wavelength of the decomposition wave agrees within a factor of 2 with the estimated value and the experimentally determined macroscopic magnetic and ME characteristics of the PZT/ NFO composite agree reasonably well with those estimated theoretically for the underlying nanostructure. It appears that the method described in this letter can serve to synthesize a number of functional composites.
The support of the National Science Foundation, Grant No. DMR0354740, and the Office of Naval Research, Contract No. N000140110761 is acknowledged. The authors are also grateful to I. Lloyd for her hospitality, S. Lofland, and the UMD-MRSEC program, DMR-00-0520471 for permission to use his/its equipment.

${ }^{1}$ L. D. Landau, E. M. Lifshitz, and A. L. King, Am. J. Phys. 29, 647 (1961).

${ }^{2}$ J. Van Suchetelen, Philips Res. Rep. 27, 28 (1972).

${ }^{3}$ R. Ramesh and N. A. Spaldin, Nat. Mater. 6, 21 (2007).

${ }^{4}$ M. Fiebig, J. Phys. D 38, R1 (2005).

${ }^{5}$ N. A. Spaldin and M. Fiebig, Science 309, 391 (2005).

${ }^{6}$ F. A. Smolenski and I. E. Chupis, Sov. Phys. Usp. 25, 475 (1982).

${ }^{7}$ W. Eerenstein, N. D. Mathur, and J. F. Scott, Nature (London) 442, 759 (2006).

${ }^{8}$ K. Mori and M. Wuttig, Appl. Phys. Lett. 81, 100 (2002).

${ }^{9}$ S. X. Dong, J.-F. Li, and D. Viehland, Appl. Phys. Lett. 83, 2265 (2003).

${ }^{10}$ S. X. Dong, J. Y. Zhai, N. G. Wang, F. M. Bai, J.-F. Li, D. Viehland, and T. A. Lograsso, Appl. Phys. Lett. 87, 222504 (2005).

${ }^{11}$ R. V. Chopdekar and Y. Suzuki, Appl. Phys. Lett. 89, 182506 (2006).

${ }^{12}$ S. Stein, M. Wuttig, D. Viehland, and E. Quandt, J. Appl. Phys. 97, 10Q301 (2005).

${ }^{13}$ M. A. Zurbuchen, T. Wu, S. Saha, J. Mitchell, and S. K. Streiffer, Appl. Phys. Lett. 87, 232908 (2005).

${ }^{14}$ D. Dale, A. Fleet, J. D. Brock, and Y. Suzuki, Appl. Phys. Lett. 82, 3725 (2003).

${ }^{15}$ J. Zhai, S. X. Dong, Z. Xing, J. F. Li, and D. Viehland, Appl. Phys. Lett. 89, 083507 (2006).

${ }^{16}$ S. Dong, J. Zhai, J. Li, and D. Viehland, Appl. Phys. Lett. 89, 252904 (2006).

${ }^{17}$ H. Zheng, Q. Zhan, F. Zavaliche, M. Sherburne, F. Straub, M. P. Cruz, Long-Qing Chen, U. Dahmen, and R. Ramesh, Nano Lett. 6, 1401 (2006).

${ }^{18}$ K. S. Chang, M. A. Aronova, C. L. Lin, M. Murakami, M. H. Yu, J. Hattrick-Simpers, O. O. Famodu, S. Y. Lee, R. Ramesh, M. Wuttig, I. Takeuchi, C. Gao, and L. A. Bendersky, Appl. Phys. Lett. 84, 3091 (2004).

${ }^{19}$ H. Zheng, J. Wang, S. E. Lofland, Z. Ma, L. Mohaddes-Ardabili, T. Zhao, L. Salamanca-Riba, S. R. Shinde, S. B. Ogale, F. Bai, D. Viehland, Y. Jia, D. G. Schlom, M. Wuttig, A. Roytburd, and R. Ramesh, Science 303, 661 (2004).

${ }^{20}$ C. W. Nan, G. Liu, Y. Lin, and H. Chen, Phys. Rev. Lett. 94, 197203 (2005).

${ }^{21}$ H. Zheng, J. Wang, L. Mohaddes-Ardabili, M. Wuttig, L. Salamanca-Riba, D. G. Schlom, and R. Ramesh, Appl. Phys. Lett. 85, 2035 (2004).

${ }^{22}$ J. van den Boomgaard and R. A. J. Born, J. Mater. Sci. 13, 1538 (1978).

${ }^{23}$ M. L. Calzada and S. J. Milne, J. Mater. Sci. Lett. 12, 1221 (1993).

${ }^{24}$ J. W. Cahn, J. Appl. Phys. 34, 358 (1963).

${ }^{25}$ M. Takahashi, J. R. Guimarae, and M. E. Fine, J. Am. Ceram. Soc. 54, 291 (1971).

${ }^{26}$ A. H. Morrison and K. Haneda, J. Appl. Phys. 52, 2496 (1981).

${ }^{27}$ M. T. Kim, Thin Solid Films 283, 12 (1996).

${ }^{28}$ R. M. Bozorth, E. F. Tilden, and A. J. Williams, Phys. Rev. 99, 1788 (1955).

${ }^{29}$ G. Srinivasan, E. T. Rasmussen, and R. Hayes, Phys. Rev. B 67, 014418 (2003).

${ }^{30}$ H. Grimmer, Acta Crystallogr., Sect. A: Found. Crystallogr. A48, 266 (1992). 